\title{
Supervisão e controlo de um sistema de canais de rega. Parte II - Calibração hidráulica
}

\author{
Supervision and control of an irrigation canal system. \\ Part II - Hydraulic tuning \\ Manuel Rijo $^{1 *}$, Carlos Miranda Rodrigues ${ }^{2 *}$ \\ 'Prof. Assoc. c/ Agregação Universidade de Évora, membro da APRH no732 \\ 2 Prof. Aux. Universidade de Évora \\ * Escola de Ciência e Tecnologia, Universidade de Évora, Instituto de Ciências Agrárias e Ambientais Mediterrânicas, Apartado 94, 7002-554 Évora, Portugal
}

RESUMO: O artigo faz uma apresentação breve do sistema de supervisão e controlo (SCADA) desenvolvido para as redes primária e secundária de canais do Aproveitamento Hidroagrícola de Idanha-a-Nova, já instalado e calibrado, dando uma atenção muito especial ao desenvolvimento e calibração dos controladores de caudal para comportas associadas ou não a soleiras descarregadores.

Os controladores de caudal desenvolvidos para o SCADA permitem o cálculo do caudal para todas as situações de funcionamento das comportas, garantindo a continuidade das soluções nas passagens do escoamento tipo descarregador para tipo comporta e da situação de ressalto livre para ressalto parcial ou totalmente afogado, em ambos os sentidos. O coeficiente teórico de vazão usado para iniciar o algoritmo de vazão geral foi ajustado no campo com recurso a estimativas de caudal realizadas com dois tipos de caudalímetros e para duas aberturas diferentes de cada comporta e/ou dois regimes permanentes para cada uma das instalações.

Foram usados dois tipos de caudalímetros, ambos transdutores acústicos baseados no efeito de Doppler, um fixo no rasto dos canais e o outro portátil e montado numa plataforma flutuante. $O$ artigo faz também uma apresentação breve dos caudalímetros usados, apresenta os resultados das medições de caudal efetuadas e ainda os valores corrigidos para os coeficientes de vazão.

Palavras-chave: canais de rega; tecnologia SCADA; equações de vazão; controladores de caudal; caudalímetros de efeito Doppler

ABSTRACT: The paper makes a brief presentation of the supervisory control and data acquisition system (SCADA) developed for the main and secondary canals network of the Idanha-a-Nova Irrigation District, already installed and tuned, giving a special attention to the development and field tuning of the flow controllers for the gates associated or not to weirs.

The flow controllers developed for the SCADA allow the flow computation for all the operational conditions of the gates, ensuring the solution continuity in the passages of the flow from gate to weir type and/or from free to partial or total submerged flow in one way or another. The theoretical discharge coefficient used to start the general flow algorithm was been adjusted in the field using flow computations made with two types of flowmeters and for two different openings for each gate and/or two different steady flows for each gate installation. Were used two types of flowmeters, both acoustic transducers based on the principle called the Doppler shift, one fixed on the canals bottom and the other mounted on a small moving floating platform. The paper also makes a brief presentation of the used flowmeters, presents the flow measurement results and also the corrected values for the discharge coefficients.

Keywords: irrigation canals; SCADA technology; flow equations; flow controllers; acoustic Doppler velocity meters

O texto deste artigo foi submetido para revisão e possível publicação em agosto de 2016, tendo sido aceite pela Comissão de Editores Científicos Associados em setembro de 2016. Este artigo é parte integrante da Revista Recursos Hídricos, Vol. 37, № 2, 65-78, outubro de 2016.

○ APRH, ISSN 0870-1741 | DOI 10.5894/rh37n2-cti3 


\section{INTRODUÇÃO}

Praticamente todos os canais dos Aproveitamentos Hidroagrícolas Nacionais estão equipados com o controlo local por montante, em que o controlador é uma ou mais comportas AMP ou descarregadores do tipo bico de pato (Rijo, 2010; Rijo et al., 2016).

A variável controlada no controlo local por montante é a altura de água a jusante de cada trecho de canal ou imediatamente a montante de cada controlador (comporta ou descarregador). Quando há variação de caudal em circulação e no caso de o controlador ser uma comporta, esta ajusta a sua abertura para repor a variável controlada no seu valor de referência. $O$ valor de referência é, por norma, a altura de água em regime uniforme para o caudal de dimensionamento do trecho de canal a montante da comporta, com um acréscimo para fazer face à perda de carga exigida pela comporta (por regra, sempre muito pequena).

O controlo local por montante visa apenas o controlo de alturas de água no interior dos canais. Não permite o controlo de caudais. Por isso, este tipo de controlo exige a ação complementar de um controlo de caudais, sempre manual, em todas as admissões aos canais.

Atendendo à dinâmica hidráulica de cada trecho de canal (Rijo et al. 2016), o controlo local por montante adequa-se bem às variações de caudal a montante (admissão ao trecho) e responde de forma bastante ineficiente às variações de caudal a jusante (seção de jusante do trecho ou nas tomadas de água).

Os ajustamentos (manuais) de caudal nas admissões respondem de modo muito ineficiente às variações de caudal nas tomadas de água, mesmo quando estas são conhecidas com antecipação suficiente. É impossível definir um hidrograma de caudais à cabeça de um canal que satisfaça a distribuição de água em todas as tomadas de água de forma otimizada. Quando as variações de caudal nas tomadas são imprevisíveis e até de sinais contrários, a ineficiência do sistema aumenta extraordinariamente, dando origem a grandes perdas ou falta de água, sobretudo nas zonas de jusante dos canais (Rijo, 2010).

Ossistemas SCADA (acrónimo inglês que significa Supervisory Control And Data Acquisition) permitem a monitorização e telecomando de órgãos hidráulicos em tempo real. Por isso, têm vindo a ser usados, cada vez mais, mesmo a nível nacional, para melhorar a gestão dos canais com controlo por montante (Rijo et al., 2016). O controlo de caudais nas admissões aos canais passa a ser realizado por telecomando em tempo real e a monitorização do estado hidráulico passa também a ser possível em tempo real, ganhando-se tempo, permitindo a gestão quantificada (na gestão tradicional, na maioria das vezes, nem sequer é possível calcular os caudais admitidos) e reduzindo as perdas de água associadas ao controlo.

Rijo et al. (2016) faz a apresentação geral do SCADA desenvolvido e implementado para a modernização das redes primária e secundária de rega do Aproveitamento Hidroagrícola de Idanha-a-Nova (AHIN).

O presente artigo faz a apresentação dos controladores de caudal para as comportas integrados nesse SCADA, do algoritmo geral para cálculo da vazão nessas comportas e da sua calibração de campo. Apresenta ainda a avaliação de campo dos caudalímetros instalados para a supervisão de caudais em tempo real pelo SCADA.

\section{BREVE APRESENTAÇÃO DO SCADA DO AHIN}

O SCADA dos canais primários e secundários do AHIN foi instalado e calibrado durante o ano de 2014. A fase última de calibração decorreu no final da campanha de rega desse ano.

Os canais primários e secundários do AHIN são apresentados e caracterizados em Rijo et al. (2016).

O SCADA é, basicamente, constituído pelos seguintes elementos:

- Centro de comando e supervisão - para o comando e a supervisão dos órgãos hidráulicos principais e a supervisão das alturas de água nas secções escolhidas;

- Unidades Locais (Estações de Campo) - para a recolha de informação local e o comando dos órgãos hidráulicos, obedecendo às ordens do centro de comando e supervisão ou trabalhando de modo independente;

- Sistema de comunicações - para a troca de dados e de informação entre o centro de comando e supervisão e as unidades locais. 
O centro de comando e supervisão está instalado na sede da ARBI-Associação de Regantes e Beneficiários de Idanha-a-Nova, que tem a responsabilidade da gestão do AHIN. A Figura 1 e a Tabela 1 apresentam a localização e tipologia das 16 estações de campo projetadas e instaladas.

As unidades de campo do SCADA são de dois tipos:

- ULcm - Unidade local com controlo (comando ou atuação) e monitorização (supervisão ou telemetria); possui equipamento para telecomando, função de data logging e posterior envio dos dados guardados para o centro de comando e supervisão e pode reunir as funções que seguem:

monitorização de alturas de água - a montante $\left(h_{1}\right)$ ou a jusante $\left(h_{2}\right)$ de um órgão ou estrutura hidráulica (limpa grelhas, comporta, descarregador frontal ou lateral);

monitorização de aberturas de comportas (a); monitorização de caudais para o sistema de drenagem através de descarregador $\left(Q_{1}\right)$ e/ou com recurso a caudalímetro $\left(Q_{2}\right)$; controlo de caudais $(Q)$ - de admissão a distribuidor, a reservatório ou de saída através de comporta.

- ULm - Unidade local de monitorização (supervisão ou telemetria); não possui equipamento para telecomando, tendo apenas a função de data logging e posterior envio dos dados guardados para o centro de comando e supervisão, com uma ou mais das funções que seguem:

> monitorização de alturas de água - a montante $\left(h_{1}\right)$ ou a jusante $\left(h_{2}\right)$ de uma máquina, órgão ou estrutura hidráulica (limpa grelhas, comporta, descarregador frontal ou lateral);

> monitorização deaberturas decomportas (a);

monitorização de caudais para o sistema de drenagem através de descarregador $\left(Q_{1}\right)$ e/ ou com recurso a caudalímetro $\left(Q_{2}\right)$.

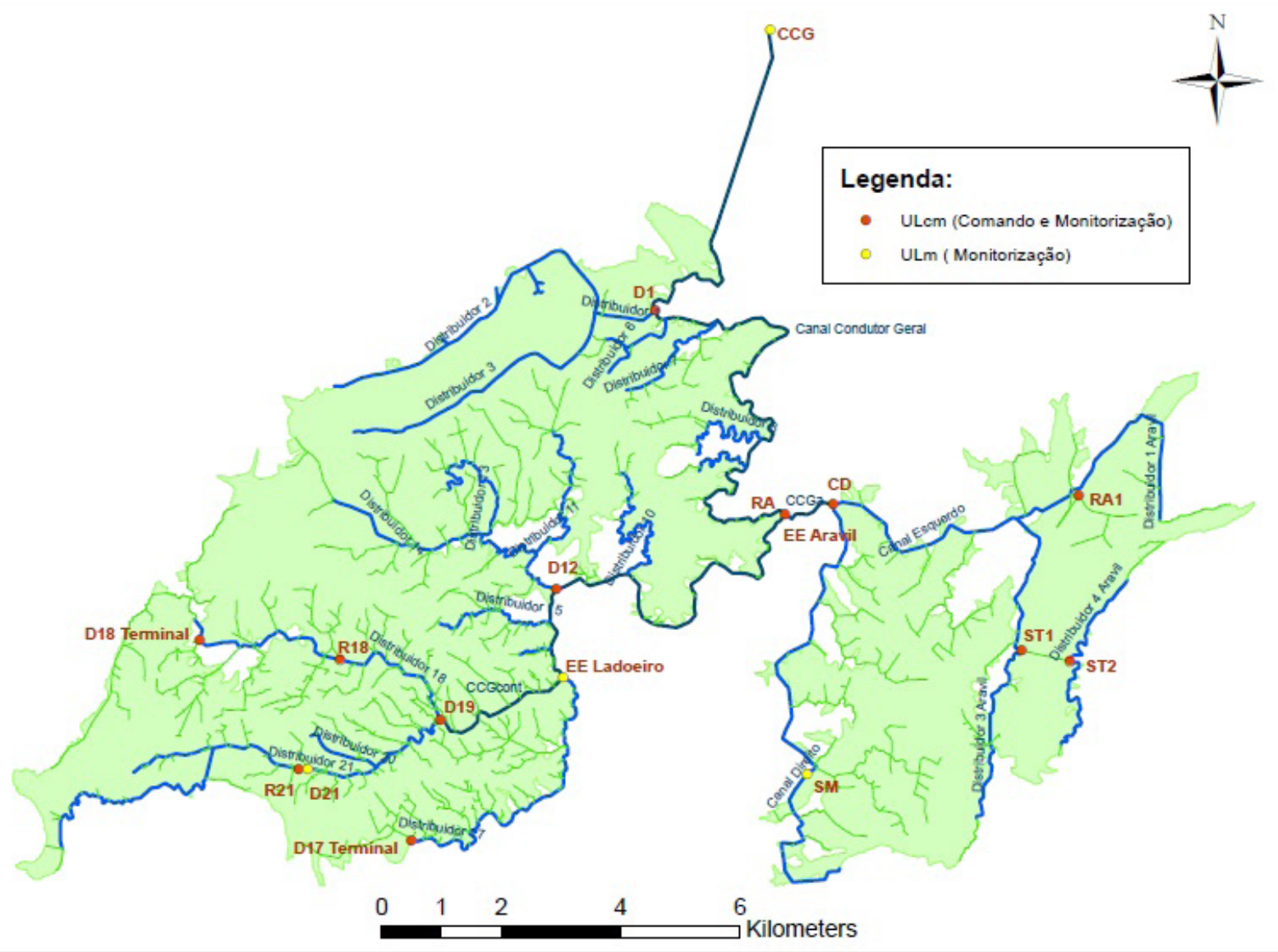

Figura 1. Estações de campo do SCADA das redes primária e secundária do AHIN (Rijo et al., 2016). 
Tabela 1. Caracterização sumária das estações de campo do SCADA do AHIN (Rijo et al., 2016).

\begin{tabular}{|c|c|c|c|c|c|c|c|c|c|}
\hline Estação & $\begin{array}{l}\text { Localização } \\
\text { da estação }\end{array}$ & $\begin{array}{c}\text { Tipo } \\
\text { estação }\end{array}$ & $\begin{array}{c}\text { No } \\
\text { comportas }\end{array}$ & $\begin{array}{l}\text { Ressalto } \\
\text { hidráulico }\end{array}$ & Monitorização & Descarregadores & $\begin{array}{l}\text { Sensores } \\
\text { caudal }\end{array}$ & Controladores & Energia \\
\hline \multicolumn{10}{|c|}{ Canal Condutor Geral } \\
\hline CCG & Admis. CCG & ULm & --- & --- & $(3 *)$ & -- & --- & --- & Rede \\
\hline D1 & $\begin{array}{l}\text { Admis. } \\
\text { Distribuidor } \\
1\end{array}$ & ULcm & 1 & $\begin{array}{l}\text { Livre/ } \\
\text { subm. }\end{array}$ & $h_{l}, h_{2}, a$ & --- & -- & $D, P, Q$ & $\begin{array}{l}\text { Painel } \\
\text { solar }\end{array}$ \\
\hline $\begin{array}{l}\text { RA-EE } \\
\text { Aravil }\end{array}$ & EE Aravil & ULcm & $1\left(^{*}\right)$ & $\begin{array}{l}\text { Livre/ } \\
\text { subm. }\end{array}$ & $h_{l}, h_{2}, a, Q_{l}, Q_{2}$ & 2 & 1 & $D, P, Q\left({ }^{*}\right)$ & Rede \\
\hline D12 & $\begin{array}{l}\text { Admis. } \\
\text { Distribuidor } \\
12\end{array}$ & ULcm & 2 & $\begin{array}{l}\text { Livre/ } \\
\text { subm. }\end{array}$ & $h_{1} h_{2}, a$ & --- & --- & $D, P, Q$ & Rede \\
\hline $\begin{array}{l}\text { EE } \\
\text { Ladoeiro }\end{array}$ & EE Ladoeiro & ULm & --- & --- & $h_{l}, h_{2}, Q_{l}, Q_{2}$ & 2 & 1 & --- & Rede \\
\hline $\begin{array}{l}\text { D17 } \\
\text { Terminal }\end{array}$ & $\begin{array}{l}\text { Admis. } \\
\text { Distribuidor } \\
17\end{array}$ & ULcm & 1 & Livre & $h_{l}, a, Q_{1}$ & 2 & --- & $D, P, Q$ & $\begin{array}{l}\text { Painel } \\
\text { solar }\end{array}$ \\
\hline \multicolumn{10}{|c|}{$\begin{array}{l}\text { Canal Condutor Geral } \\
\text { continuação }\end{array}$} \\
\hline $\mathrm{R} 18$ & $\begin{array}{l}\text { Reservatório } \\
18\end{array}$ & ULcm & $\begin{array}{c}2\left(^{*}\right)+1\left(^{*}\right) \\
\left({ }^{* *}\right)\end{array}$ & $\begin{array}{l}\text { Livre/ } \\
\text { subm. }\end{array}$ & $h_{l}, h_{2}, a, Q_{l}, Q_{2}$ & 1 & $1+1\left({ }^{4 *}\right)$ & $D, P, Q$ & Rede \\
\hline $\begin{array}{l}\text { D18 } \\
\text { Terminal }\end{array}$ & $\begin{array}{l}\text { Termin. } \\
\text { Distribuidor } \\
18\end{array}$ & ULcm & 1 & $\begin{array}{l}\text { Livre/ } \\
\text { subm. }\end{array}$ & $h_{1}, h_{2}, a$ & 1 & --- & $D, P, Q$ & $\begin{array}{l}\text { Painel } \\
\text { solar }\end{array}$ \\
\hline D19 & $\begin{array}{l}\text { Distribuidor } \\
19\end{array}$ & ULcm & 1 & $\begin{array}{l}\text { Livre/ } \\
\text { subm. }\end{array}$ & $h_{1}, h_{2}, a, Q_{2}$ & -- & 1 & $D, P, Q$ & Rede \\
\hline D21 & $\begin{array}{l}\text { Distribuidor } \\
21\end{array}$ & ULm & --- & --- & $h_{1}, Q_{2}$ & -- & 1 & --- & $\begin{array}{l}\text { Painel } \\
\text { solar }\end{array}$ \\
\hline $\mathrm{R} 21$ & $\begin{array}{l}\text { Reservatório } \\
21\end{array}$ & ULcm & $1\left(^{*}\right)\left({ }^{* *}\right)$ & --- & $h_{l}, a, Q_{l}$ & 1 & --- & $D, P, Q$ & Rede \\
\hline \multicolumn{10}{|c|}{$\begin{array}{l}\text { Canal Condutor Geral } \\
\text { do Aravil }\end{array}$} \\
\hline$C D$ & $\begin{array}{l}\text { Admis. } \\
\text { Canal } \\
\text { Direito }\end{array}$ & ULcm & 2 & $\begin{array}{l}\text { Livre/ } \\
\text { subm. }\end{array}$ & $h_{1}, h_{2}, a$ & -- & --- & $D, P, Q$ & Rede \\
\hline SM & $\begin{array}{l}\text { Sifão } \\
\text { Mascarenha }\end{array}$ & ULm & --- & --- & $h_{l}, Q_{l}, Q_{2}$ & 1 & 1 & --- & $\begin{array}{l}\text { Painel } \\
\text { solar }\end{array}$ \\
\hline RA1 & $\begin{array}{l}\text { Reservatório } \\
1 \text { do Aravil }\end{array}$ & ULcm & $\begin{array}{c}3(*)+1\left(^{*}\right) \\
\left({ }^{* *}\right)\end{array}$ & $\begin{array}{l}\text { Livre/ } \\
\text { subm. }\end{array}$ & $h_{1}, h_{2}, a, Q_{2}$ & 1 & --- & $D, P, Q$ & Rede \\
\hline ST1 & $\begin{array}{l}\text { Admissão } \\
\text { Sifão Toula }\end{array}$ & ULcm & 1 & Livre & $h_{l}, a, Q_{l}$ & 1 & --- & $D, P, Q$ & $\begin{array}{l}\text { Painel } \\
\text { solar }\end{array}$ \\
\hline ST2 & $\begin{array}{l}\text { Saída Sifão } \\
\text { Toula }\end{array}$ & ULcm & $1+1$ & $\begin{array}{l}\text { Livre/ } \\
\text { subm. }\end{array}$ & $h_{1}, h_{2}, a$ & --- & --- & $D, P, Q$ & $\begin{array}{l}\text { Painel } \\
\text { solar }\end{array}$ \\
\hline
\end{tabular}

(*) Já instalada

(**) Comporta mural da tomada de água; escoamento em pressão

(3*) Leitura de sinais de SCADA já existente.

$\left({ }^{4} *\right)$ Medidor de caudal eletromagnético na conduta da tomada de água

$\left({ }^{5 *}\right) \quad$ Com possibilidade de inversão do caudal 


\section{CONTROLADOR DIGITAL DE CAUDAIS DO SCADA PARA COMPORTAS}

A Tabela 1 apresenta o controlador direto para uma ou mais comportas ( $D$, Tabela 1$)$, que consiste na capacidade de um utilizador através de uma $H M I$ - acionar um botão para a abrir, fechar ou parar numa determinada posição. Estas ações de comando podem ser arbitrárias (decisão do operador humano) ou resultar de um controlador automático.

A mesma tabela apresenta ainda o controlador de posição para uma ou mais comportas, que permite ajustar a posição da(s) comporta(s) e que consiste na capacidade de envio de uma ordem de comando (Abrir/Fechar/Stop) a transmitir ao controlador direto $(P$, Tabela 1$)$. As saídas do controlador de posição vão ser as entradas do controlador direto.

A Tabela 1 apresenta também o controlador de caudal para uma ou mais comportas $(Q$, Tabela 1). Associado ao algoritmo de caudal, existe um controlador direto responsável pelo envio da ordem de comando para o atuador elétrico da comporta.

A Figura 2 apresenta o controlador de caudal para duas comportas. O controlador recebe a referência ou ordem de caudal $Q_{r}$, caudal total para as duas comportas. Tendo em conta as alturas de águas a montante e a jusante das comportas, $h_{1}$ e $h_{2^{\prime}}$ e as aberturas das mesmas, $a_{\text {Imed }}$ e $a_{2 m e d}$, o controlador calcula o caudal $Q$, caudal atual ou que está a passar naquela situação nas duas comportas.

O cálculo do desvio $\left(e_{Q}\right)$ entre o caudal atual $(Q$, dado pela equação de vazão da comporta) e o novo caudal $\left(Q_{r}\right)$ pode originar três situações distintas:

- Se $e_{Q}>\Delta \mathrm{Q}$ (erro positivo: necessidade de aumentar o caudal) - Abre comportas (as duas em simultâneo na situação normal, ou, havendo avaria numa delas, a outra deverá compensar a diferença de abertura desta);

- Se $e_{Q}<\Delta \mathrm{Q}$ (erro negativo: necessidade de diminuir o caudal) - Fecha comportas;

- Se $e_{Q}=0$ (erro nulo: necessidade de manter o caudal) - Imobiliza comportas.

A saída do controlador de caudal - $a_{1 r}$ e $a_{2 r}$ representa as ordens de abrir/fechar comportas transmitidas ao controlador direto.

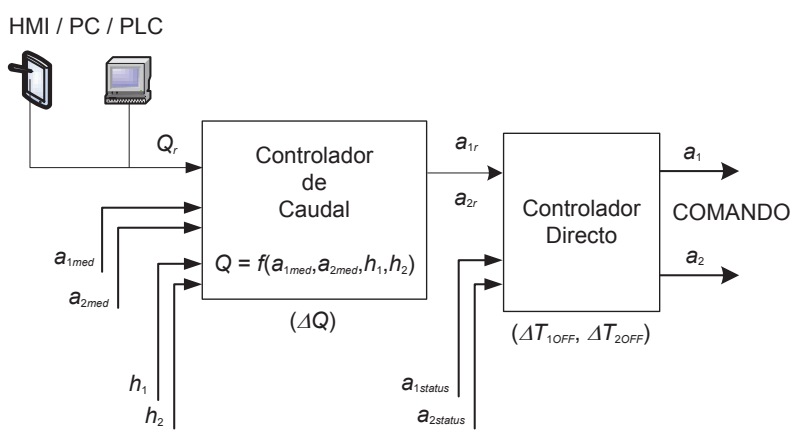

Figura 2. Controlador de caudal para duas comportas (Rijo, 2010).

Cálculo do caudal $Q$ no controlador de caudal- As estruturas de controlo de caudais nas admissões aos canais podem funcionar como descarregadores (comportas acima da superfície livre) ou como comportas associadas a soleiras e, para cada situação, haver ainda situações de escoamento livre (ressalto hidráulico livre a jusante), escoamento parcialmente submerso e escoamento totalmente submerso (ressalto hidráulico a jusante com submersão parcial ou total).

As equações de vazão a usar para cada estrutura e cada situação de escoamento são bem conhecidas. Porém, na passagem de uma para outra equação, raramente existe continuidade numérica nas respetivas soluções, o que dificulta a definição de algoritmos gerais de vazão para programação dos autómatos.

O algoritmo geral de cálculo de $Q$ usado nos autómatos garante a continuidade das soluções para todo o domínio de funcionamento das estruturas, não havendo lugar a descontinuidades numéricas na passagem de, por exemplo, descarregador para comporta ou de escoamento livre para submerso num ou noutro sentido. As equações usadas garantem os mesmos valores na região de fronteira (Rijo et al., 2007).

O algoritmo geral foi definido por Baume et al. (2000).

Considere-se o esquema geral da Figura 3, que representa uma comporta associada a uma soleira baixa ( $p_{1} \rightarrow 0$ e $p_{2} \rightarrow 0$ ), e considere-se a equação geral de vazão do descarregador controlado por comporta (Figura 3). 


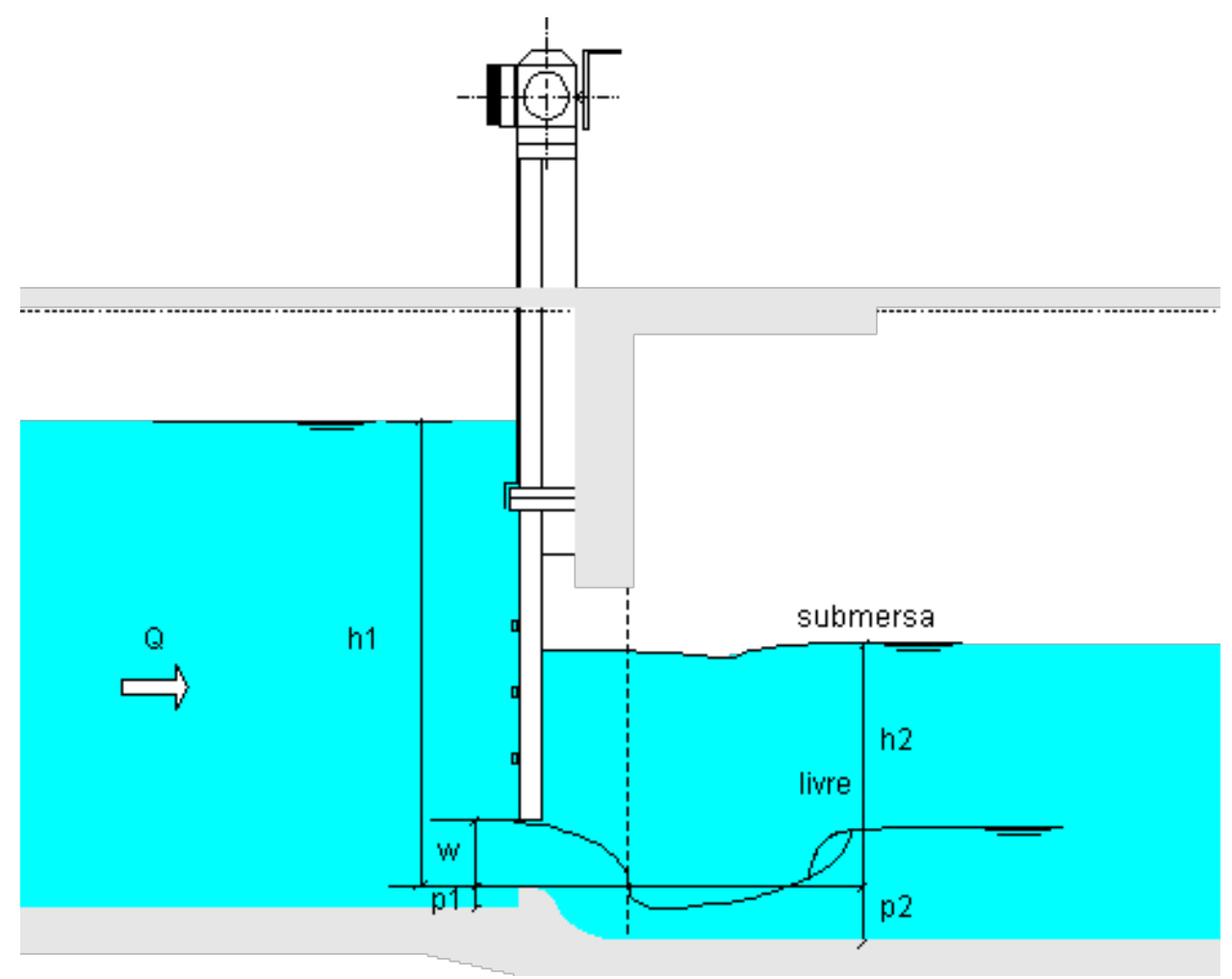

Figura 3. Comporta associada a soleira baixa (Rijo et al., 2007).

$$
Q=l \sqrt{2 g}\left[k_{a} h_{1}^{3 / 2}-k_{b}\left(h_{1}-w\right)^{3 / 2}\right]
$$

em que $l$ é o comprimento do descarregador (ou largura da comporta), g é a aceleração da gravidade e em que $k_{a}=k_{F} \mu$ e $k_{b}=k_{F 1} \mu_{1}$, sendo $k_{F}$ e $k_{F I}$ coeficientes de redução e $\mu$ e $\mu_{1}$ coeficientes de vazão para a situação de escoamento livre. O escoamento é do tipo:

- descarregador, quando_ $w \geq h_{1}$ e $k_{b}=0$;

- comporta, quando $w\left\langle h_{1}\right.$.

O algoritmo geral de vazão, correspondente à equação (1), usado diretamente na programação dos controladores de caudal nos autómatos apresenta-se na Figura 4 (na figura, a variável a representa a altura de abertura da comporta, também representada no texto por $w$ ).

\section{CALIBRAÇÃO DAS EQUAÇÕES DE VAZÃO DAS COMPORTAS}

\subsection{Metodologia de campo usada}

A metodologia seguida no campo para calibração e ajustamento do algoritmo geral de vazão programado em cada autómato foi a seguinte:

- verificar se as diferentes escalas limnimétricas instaladas para calibração dos sensores de nível estavam ou não referidas à crista das soleiras associadas às comportas ou ao rasto do canal, nas situações em que não havia soleira;

- ajustar os sensores de nível (alturas de água) tendo em conta as referências do ponto anterior;

- conferir a largura útil das comportas;

- conferir os fins de curso das comportas;

- fazer estimativa de caudais com um caudalímetro portátil do tipo Survey River Sontek S5 (SRS5) (SonTek 2010) em regime permanente para duas situações de funcionamento diferentes (aberturas de comporta diferentes) e/ou regimes de escoamento permanentes diferentes em cada instalação;

- registar os valores do autómato - alturas de água, posição (ões) de comporta(s) e caudal - correspondentes a cada medição com o caudalímetro SRS5, 


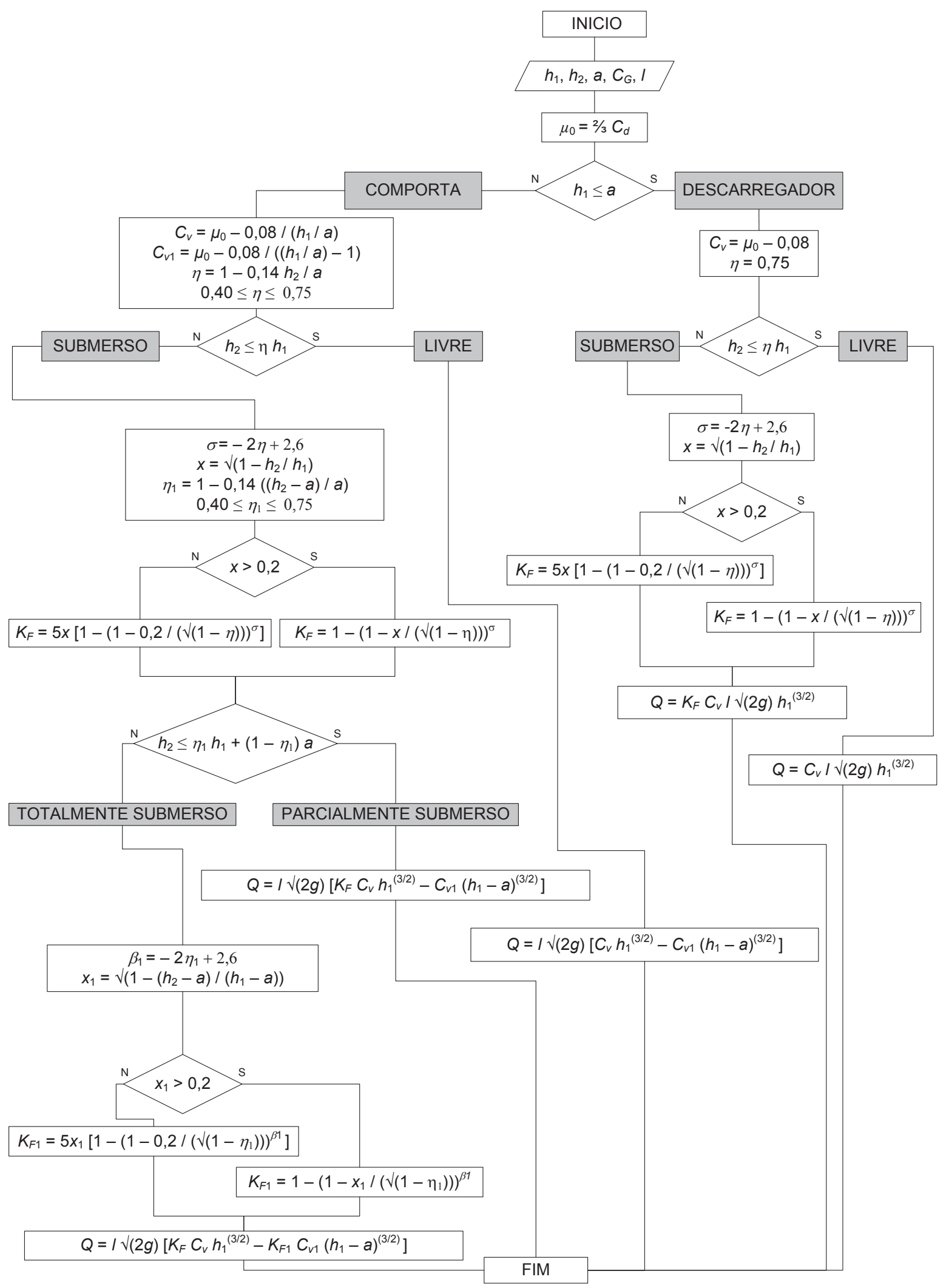

Figura 4. Cálculo do caudal em soleiras baixas controladas ou não por comportas (Rijo, 2010). 
de modo a avaliar a programação do autómato e, eventualmente, ajustar o coeficiente teórico do algoritmo de vazão programado; quando possível e necessário, as estimativas de caudal foram também realizadas recorrendo à informação de caudalímetros fixos do tipo Sontek IQ (SIQ) (SonTek 2012) instalados nas proximidades.

\subsection{Caudalímetros usados}

\section{Caudalímetros fixos}

Os caudalímetros instalados são equipamentos do tipo vulgarmente conhecido por ADVM (Acoustic Doppler Velocity Meters) que, recorrendo ao efeito de Doppler para medir a velocidade da corrente, possibilitam a determinação da vazão através do método secção - velocidade.

Instalado no rasto do canal, o caudalímetro SIQ determina a profundidade e a velocidade média na secção transversal, recorrendo à emissão de cinco feixes acústicos. Um sensor de pressão e um feixe acústico vertical são usados em conjunto para determinar o nível de água (altura de água), enquanto os outros quatro sensores acústicos (transdutores) são alocados à medição da velocidade da água por efeito Doppler. Dois destes transdutores emitem feixes inclinados segundo a direção do escoamento e servem para o apuramento da velocidade média da secção líquida transversal que é constantemente corrigida dos fluxos horizontais monitorizados pelos outros dois transdutores, orientados para cada uma das margens do canal.

A Figura 5 apresenta a instalação tipo do SIQ em canais.

O caudal é determinado usando uma combinação de dados do nível de água, que são convertidos em área de secção transversal liquida através da relação $\mathrm{A}=\mathrm{f}(\mathrm{h})$ que resulta da parametrização prévia da geometria do canal. A área da secção transversal é multiplicada pela velocidade média para determinar a vazão.

As características operacionais principais do SIQ são apresentadas na Tabela 2.

A Tabela 1 assinala as estações de campo do SCADA munidas de sensores de caudal ou caudalímetros SIQ. A monitorização de caudal correspondente é assinalada na mesma tabela pela variável $Q_{2}$.

\section{Caudalímetro móvel}

A avaliação das medições dos caudalímetros fixos SIQ foi realizada recorrendo a um equipamento ADCP (Acoustic Doppler Current Profile) da marca Sontek RiverSurveyor S5

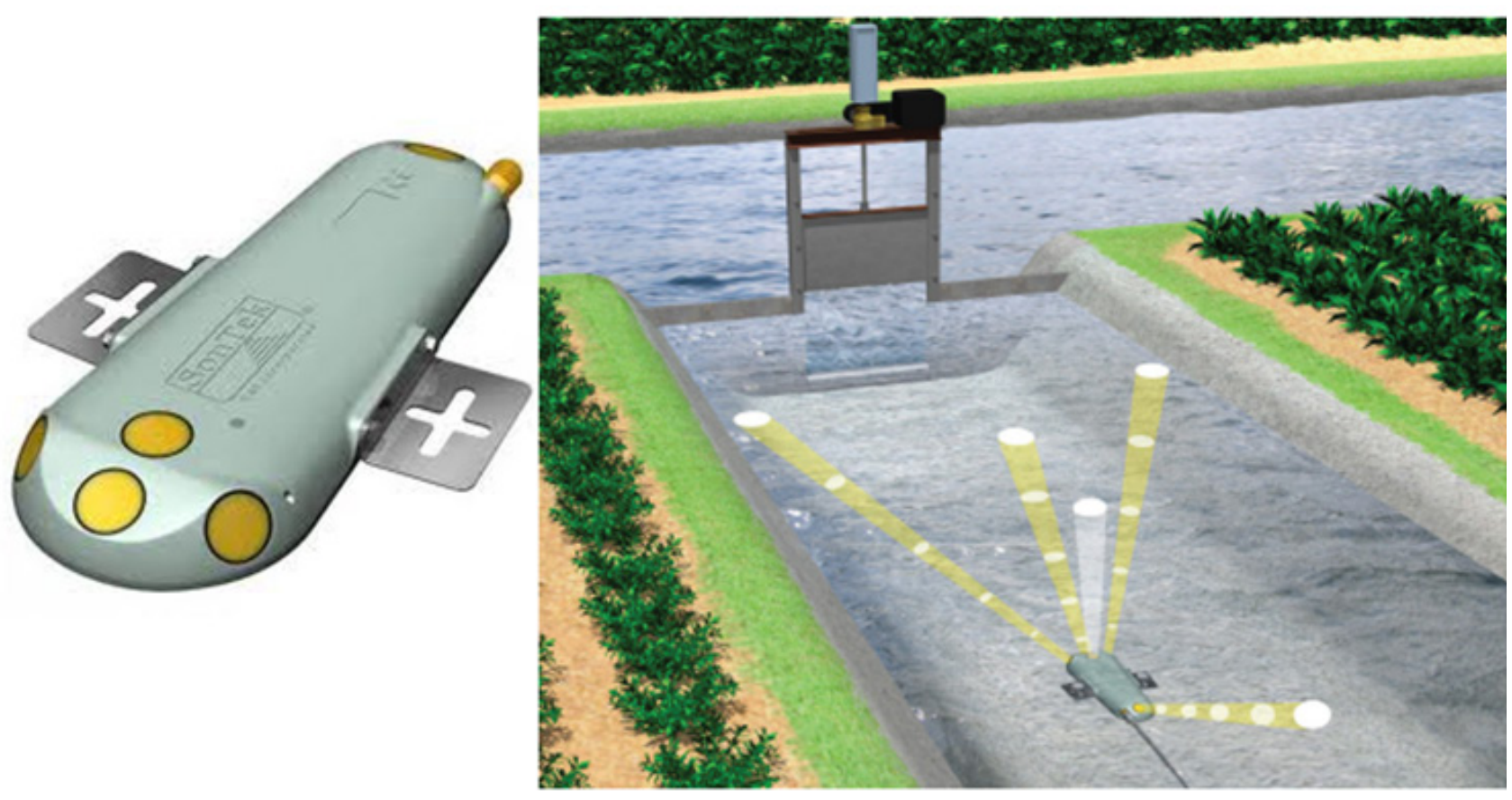

Figura 5. Sontek IQ e instalação tipo em canal (Sontek, 2012). 
Tabela 2. Principais caraterísticas operacionais do caudalímetro SIQ (SonTek, 2012).

\begin{tabular}{|c|c|c|c|c|c|c|c|c|c|}
\hline Número & \multicolumn{3}{|c|}{ Velocidade $(m / s)$} & \multicolumn{3}{|c|}{ Profundidade $(m)$} & \multicolumn{3}{c|}{ Pressão $(m)$} \\
\hline células & Gama & Resolução & Precisão & Gama & Resolução & Precisão & Gama & Resolução & Precisão \\
\hline $1 \ldots$ & $0 \ldots$ & 0,0001 & $\pm 0,005$ & $\begin{array}{c}0,05 \ldots \\
1,50\end{array}$ & 0,001 & 0,003 & $0-30$ & 0,01 & 0,03 \\
\hline 100 & & & & & & & & &
\end{tabular}

(SRS5). Tal como o SIQ, o SRS5 utiliza o efeito de Doppler para determinar a velocidade da corrente como meio para calcular o caudal na secção líquida transversal.

Basicamente, o sistema é constituídos por: uma cabeça sensora (Figura 6) que agrega um sistema de cinco feixes (transdutores acústicos) distribuídos em duas frequências diferentes - quatro deles de medição de velocidade de $3,0 \mathrm{MHz}$ e o quinto de $1,0 \mathrm{MHz}$ que funciona como um ecobatímetro fornecendo os dados de profundidade; um sistema interno de processamento dos dados, responsável pelos cálculos numéricos; uma deck box com baterias para alimentação que serve de interface entre o processador e um microcomputador através de uma ligação por cabo ou remotamente via rádio (Bluetooth).

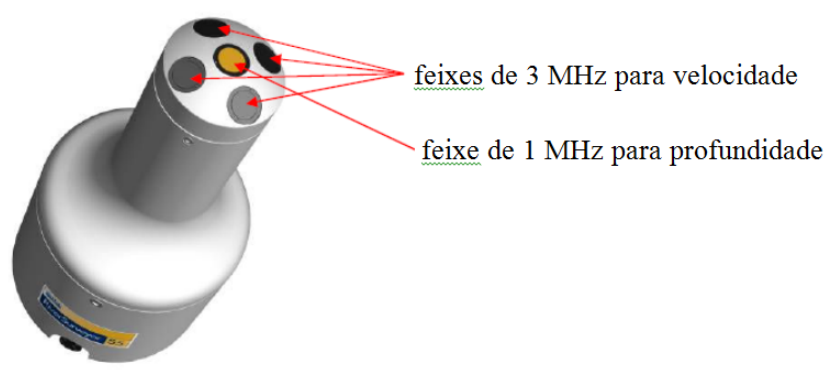

Figura 6. Cabeça sensora do caudalímetro SRS5 (Sontek, 2010).

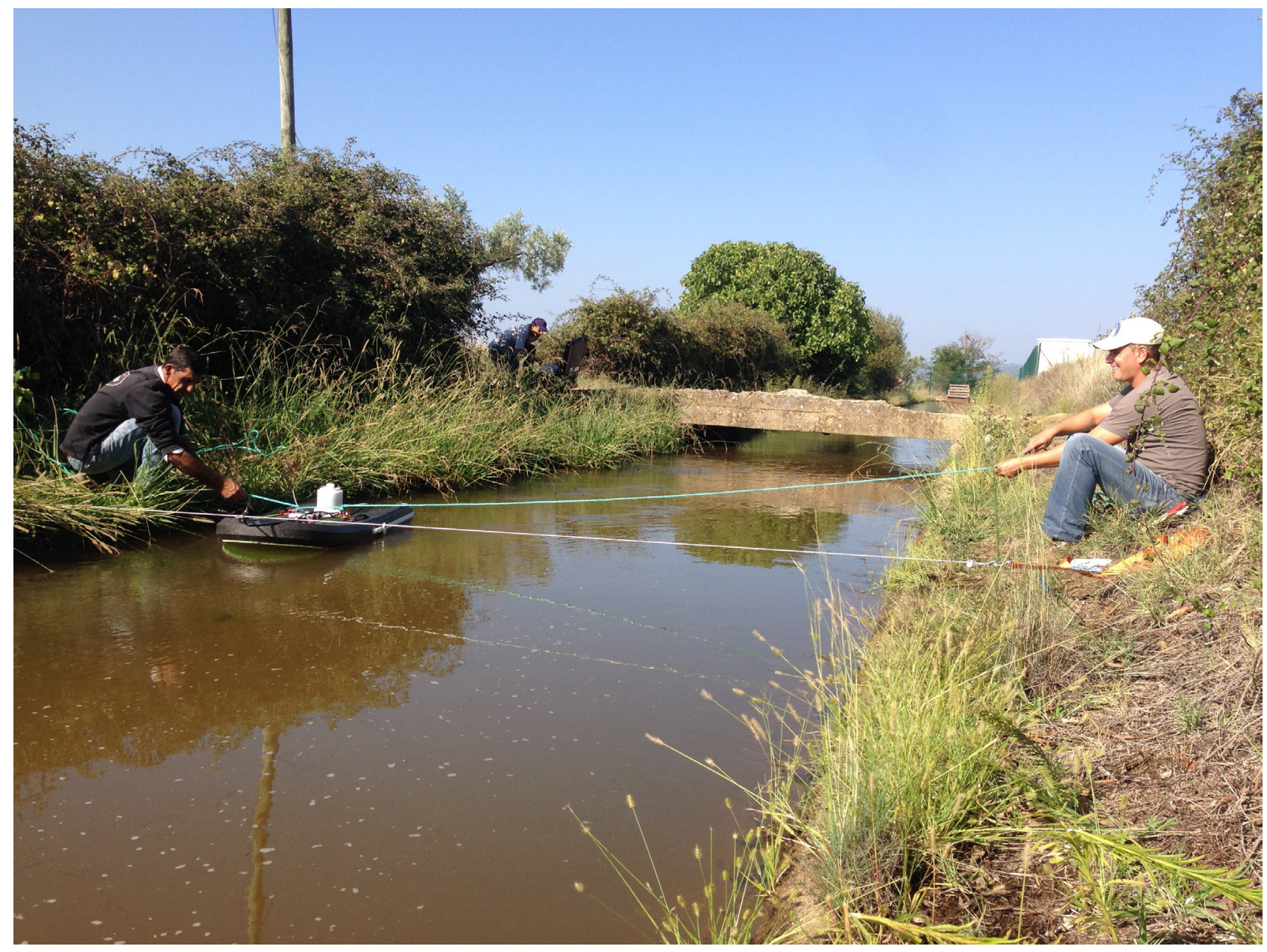

Figura 7. Medições do caudal com o SRS5 no Distribuidor D18 a montante da estação R18. 
No processo de medição, o equipamento é colocado numa prancha flutuante específica (hidroboard) que o(s) operador(es) faz(em) deslocar vezes sucessivas entre margens (Figura 7). A secção transversal é automaticamente dividida em células de dimensões $\Delta \mathrm{z}$ (profundidade) e $\Delta \mathrm{L}$ (largura) em função da velocidade de deslocamento da prancha, até um máximo de 128 células por secção. A medida da velocidade efetuada pelo ADCP é uma média sobre cada elemento de área definido por $\Delta z$ e $\Delta$ L e o caudal é calculado para cada uma das células per si e integrado ao logo da secção transversal.

O microcomputador, através do software proprietário RiverSurveyor, permite informar o sistema das parametrizações prévias à medição, nomeadamente, a profundidade dos transdutores, a declinação magnética, a distância à margem e a margem de início da determinação (direita ou esquerda). Durante a medição, o RiverSurveyor recolhe os dados, exibe-os em tempo real e armazenaos em arquivos específicos permitindo um processamento e análise posterior à medição. Entre as principais funções do programa estão: a recolha dos dados da velocidade da corrente, cálculo da área da secção medida, cálculo do caudal total, a exibição dos perfis batimétrico e de velocidades da secção, a exibição da velocidade e direção da prancha do ADCP e apresentação de indicadores estatísticos da qualidade dos dados no momento em que são colhidos.

As características operacionais principais do SRS5 são apresentadas na Tabela 3.

A diferença principal entres os dois equipamentos é que o SIQ faz a estimativa da velocidade usando apenas um perfil vertical do escoamento (nesse perfil faz até 100 estimativas pontuais da velocidade) e o SRS5 faz essa estimativa em até 128 células em toda a secção líquida, "varridas" à medida que o equipamento se desloca de uma para a outra margem. Por isso, o rigor obtido na estimativa da velocidade média do escoamento para toda a secção transversal líquida com o SRS5 é muito superior ao obtido pelo SIQ. O SRS5 é, habitualmente, usado para calibrar a equação que fornece a velocidade média do escoamento na secção transversal no caudalímetro SIQ, o chamado índice de velocidade (SonTek, 2012).

$\mathrm{O}$ índice de velocidade permite estimativas da velocidade média do escoamento para toda a secção transversal com precisões elevadas, situadas no intervalo $\pm 2 \%$ (ITRC, 2004). Os fabricantes indicam precisões de até $\pm 0,5 \%$ para o SIQ (Tabela 2 ) e de $\pm 0,2 \%$ para o SRS5, mas enquanto aquela diz respeito à velocidade média ao longo do perfil vertical do escoamento considerado, a última é definida para a velocidade média do escoamento em toda a secção transversal líquida. Considerando apenas esta última velocidade, pode dizer-se, grosso modo, que o SRS5 terá uma precisão dez vezes superior à do SIQ.

\subsection{Verificação da calibração dos caudalímetros SIQ}

Os caudalímetros SIQ instalados no AHIN foram parametrizados para estimarem o caudal através do algoritmo de cálculo fornecido pelo fabricante do equipamento. Este algoritmo baseia-se em equações teóricas de velocidade e caudal fortemente consolidadas em centenas de medições de campo para diferentes secções transversais e gama de velocidades (SonTek, 2012).

Na verificação da calibração dos SIQ foi usada como metodologia a comparação direta do caudal estimado pelo SIQ com o caudal obtido através do caudalímetro móvel SRS5.

A Tabela 4 faz a comparação dos caudais estimados pelo SIQ e pelo SRS5 em cinco estações decampo doSCADA.Osvaloresmédios

Tabela 3. Principais caraterísticas operacionais do caudalímetro SRS5 (SonTek, 2010).

\begin{tabular}{|c|c|c|c|c|c|c|c|c|c|}
\hline No Máximo & \multicolumn{3}{|c|}{ Velocidade $(m / s)$} & \multicolumn{3}{c|}{ Profundidade $(m)$} & \multicolumn{3}{c|}{ Temperatura $\left({ }^{\circ} \mathrm{C}\right)$} \\
\hline de células & Gama & Resolução & Precisão & Gama & Resolução & Precisão & gama & resolução & Precisão \\
\hline 128 & $0 \ldots 20$ & 0,001 & $\pm 0,002$ & $0,02 \ldots 15$ & 0,001 & 0,001 & $5 \ldots . .45$ & 0,01 & 0,1 \\
\hline
\end{tabular}


do SIQ traduzem a média dos valores de caudais registados em contínuo pelo caudalímetro fixo durante o período de realização das determinações com o caudalímetro móvel. Os valores médios do SRS5 correspondem à média das medições de caudal consideradas efetivas depois de expurgadas as determinações que diferiram mais de $5 \%$ da média das medições efetuadas em cada secção (assumiu-se que o nível de confiança de $95 \%$ estabelece o limite para as incertezas numa medição de caudal com o SRS5).

Os resultados obtidos permitem concluir que as medições de caudal de um e de outro equipamento são relativamente consistentes a dispersão dos respetivos resultados em torno da média, dada pelo desvio padrão (D.P., Tabela 4), para cada equipamento é relativamente baixa em todas as medições. As medições do SRS5 apresentam um valor médio superior em três secções e um valor médio inferior nas outras duas, com diferenças de, respetivamente,
$+10 \%,+2 \%,+1,5 \%,-2 \%$ e $-10 \%$ em relação às medições do SIQ.

A Figura 8 faz a comparação dos valores de caudal obtidos pelo SIQ e pelo SRS5 para as cinco estações. Na figura, é visível que uma das estações (RA-Aravil) apresenta diferenças de caudal mais significativas, podendo, eventualmente, tal facto ser explicado por os sensores do SIQ estarem sujos devido a sedimentos.

Atendendo às condições de campo, foi adotado como critério que diferenças até 10\% entre as medições dos dois equipamentos são aceitáveis e, por isso, que a parametrização do SIQ com base na equação de calibração teórica fornecida pelos fabricantes parece garantir rigor suficiente ao cálculo do caudal. Nas situações de campo, os valores obtidos pelo SIQ são muito influenciados pelo estado de limpeza das águas e das cabeças dos sensores, o que não se pode ignorar.

Tabela 4. Caudais estimados pelos caudalímetros SIQ e SRS5.

\begin{tabular}{|l|c|c|c|c|c|c|}
\hline \multirow{2}{*}{ Estação Campo } & \multicolumn{3}{|c|}{ SIQ } & \multicolumn{3}{c|}{ SRS5 } \\
\hline RA-Aravil PS & Média $\left(\mathrm{m}^{3} / \mathrm{s}\right)$ & D.P. $\left(\mathrm{m}^{3} / \mathrm{s}\right)$ & $\mathbf{N}^{\circ}$ Leituras & Média $\left(\mathrm{m}^{3} / \mathrm{s}\right)$ & D.P. $\left(\mathrm{m}^{3} / \mathrm{s}\right)$ & $\mathbf{N}^{\circ}$ Leituras \\
\hline Ladoeiro PS & 1.016 & 0.068 & 52 & 1.120 & 0.020 & 6 \\
\hline R18 & 0.858 & 0.036 & 22 & 0.839 & 0.016 & 6 \\
\hline D19 & 0.419 & 0.011 & 64 & 0.428 & 0.010 & 5 \\
\hline SM & 0.773 & 0.016 & 22 & 0.784 & 0.013 & 9 \\
\hline
\end{tabular}

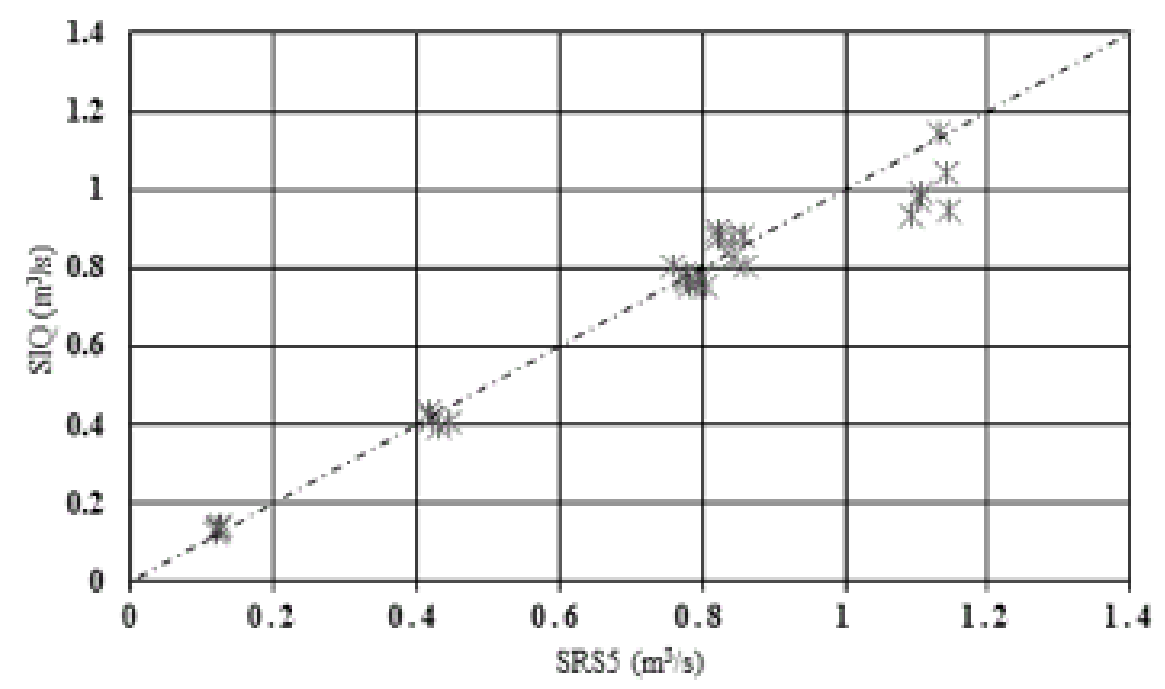

Figura 8. Comparação de caudais obtidos pelos SIQ e SRS5, para as cinco estações de campo. 


\subsection{Definição dos coeficientes de vazão das comportas}

Os resultados das calibrações de campo apresentam-se na Tabela 5. A tabela apresenta os caudais lidos nos diferentes autómatos considerando o valor teórico de 0,60 para o coeficiente de vazão $C_{G}$ (Figura 4). Os valores destes caudais foram confrontados com os valores correspondentes obtidos usando diretamente o algoritmo da Figura 4, de modo a averiguar da qualidade da programação de cada autómato. Quando não havia diferença, concluiu-se que os autómatos tinham sido bem programados.
Sempre que havia diferença significativa entre os valores de caudal obtidos pelo caudalímetro SRS5 e os correspondentes lidos no autómato, após verificação da fiabilidade da programação deste, corrigiu-se o valor de $C_{G}$ do autómato (valores da última coluna da Tabela 5), de modo a aproximar os dois valores, passando a considerar-se que o algoritmo de vazão passava a estar calibrado para todas as condições de campo da instalação.

A Figura 9 apresenta a média de três leituras efetuadas com o caudalímetro SRS5 na estação de campo do SCADA D12, a jusante das comportas. É este valor médio que se apresenta na Tabela 5.

Tabela 5. Calibração de campo das equações de vazão das comportas.

\begin{tabular}{|c|c|c|c|c|c|c|c|}
\hline \multirow[t]{2}{*}{ Estação } & \multicolumn{3}{|c|}{ Comporta $(m)$} & \multirow{2}{*}{$\begin{array}{l}\text { Ressalto } \\
\text { hidráulico }\end{array}$} & \multicolumn{2}{|c|}{ Caudal, $\mathbf{Q}\left(\mathrm{m}^{3} / \mathrm{s}\right)$} & \multirow[t]{2}{*}{ Correção do algoritmo do autómato } \\
\hline & $h_{1}$ & A & $h_{2}$ & & $C_{G}=0,60$ & SRS & \\
\hline D1 & $\begin{array}{l}0,395 \\
0,400\end{array}$ & $\begin{array}{l}0,117 \\
0,118\end{array}$ & $\begin{array}{l}0,277 \\
0,355\end{array}$ & $\begin{array}{l}\text { Subm. } \\
\text { Subm. }\end{array}$ & $\begin{array}{l}0,220 \\
0,123\end{array}$ & $\begin{array}{c}--\left(^{*}\right) \\
---\end{array}$ & Nenhuma \\
\hline $\begin{array}{l}\text { RA-EE } \\
\text { Aravil }\end{array}$ & $\begin{array}{l}1,380 \\
1,400\end{array}$ & $\begin{array}{l}0,865 \\
0,755\end{array}$ & & $\begin{array}{l}\text { Subm. } \\
\text { Subm. }\end{array}$ & $\begin{array}{l}1,112 \\
1,150\end{array}$ & $\begin{array}{l}0,927 \\
0,912\end{array}$ & $\begin{array}{l}C_{G}=0,5 ; \text { passando os caudais a ser } 0,925 \\
\text { e } 0,953 \mathrm{~m}^{3} / \mathrm{s} \text {, respetivamente. }\end{array}$ \\
\hline D12 & $\begin{array}{l}0,980 \\
0,990\end{array}$ & $\begin{array}{l}0,063 \\
0.144\end{array}$ & $\begin{array}{l}0,737 \\
0,740\end{array}$ & $\begin{array}{l}\text { Subm. } \\
\text { Subm. }\end{array}$ & $\begin{array}{l}0,203 \\
0,460\end{array}$ & $\begin{array}{l}0,213 \\
0,422\end{array}$ & $\begin{array}{l}\text { Nenhuma; valores do SRS muito } \\
\text { próximos dos do autómato }\end{array}$ \\
\hline $\begin{array}{l}\text { D17 } \\
\text { Terminal }\end{array}$ & $\begin{array}{l}0,270 \\
0,250\end{array}$ & $\begin{array}{l}0,066 \\
0,068\end{array}$ & $\begin{array}{c}--(* *) \\
---\end{array}$ & $\begin{array}{l}\text { Livre } \\
\text { Livre }\end{array}$ & $\begin{array}{l}0,054 \\
0,055\end{array}$ & $\begin{array}{l}0,053 \\
0,058\end{array}$ & $\begin{array}{l}\text { Nenhuma; valores do SRS muito } \\
\text { próximos dos do autómato }\end{array}$ \\
\hline R18 & $\begin{array}{l}1,293 \\
1,367\end{array}$ & $\begin{array}{l}0,095 \\
0,095\end{array}$ & $\begin{array}{l}0,410 \\
1,015\end{array}$ & $\begin{array}{l}\text { Subm. } \\
\text { Subm. }\end{array}$ & $\begin{array}{l}0,226 \\
0,200\end{array}$ & $\begin{array}{c}0,277\left({ }^{3 *}\right) \\
0,283\end{array}$ & $\begin{array}{l}\mathrm{C}_{\mathrm{G}}=0,75 ; \text { passando os caudais a ser } \\
0,280 \text { e } 0,240 \mathrm{~m}^{3} / \mathrm{s} \text {, respetivamente. }\end{array}$ \\
\hline $\begin{array}{l}\text { D18 } \\
\text { Terminal }\end{array}$ & $\begin{array}{l}1,077 \\
1,070\end{array}$ & $\begin{array}{l}0,138 \\
0,255\end{array}$ & $\begin{array}{l}0,750 \\
0,860\end{array}$ & $\begin{array}{l}\text { Subm. } \\
\text { Subm. }\end{array}$ & $\begin{array}{l}0,280 \\
0,356\end{array}$ & $\begin{array}{l}0,186 \\
0,312\end{array}$ & $\begin{array}{l}\mathrm{C}_{\mathrm{G}}=0,50 ; \text { passando os caudais a ser } \\
0,231 \text { e } 0,292 \mathrm{~m}^{3} / \mathrm{s} \text {, respetivamente. }\end{array}$ \\
\hline D19 & $\begin{array}{l}0,963 \\
0,968\end{array}$ & $\begin{array}{l}0,102 \\
0,377\end{array}$ & $\begin{array}{l}0,820 \\
0,850\end{array}$ & $\begin{array}{l}\text { Subm. } \\
\text { Subm. }\end{array}$ & $\begin{array}{l}0,141 \\
0,435\end{array}$ & $\begin{array}{l}0,085 \\
0,336\end{array}$ & $\begin{array}{l}\mathrm{C}_{\mathrm{G}}=0,45 ; \text { passando os caudais a ser } \\
0,096 \text { e } 0,332 \mathrm{~m}^{3} / \mathrm{s} \text {, respetivamente. }\end{array}$ \\
\hline$C D$ & $\begin{array}{l}1,480 \\
1,440\end{array}$ & $\begin{array}{l}0,200 \\
0,118\end{array}$ & $\begin{array}{l}1,050 \\
1,035\end{array}$ & $\begin{array}{l}\text { Subm. } \\
\text { Subm. }\end{array}$ & $\begin{array}{l}0,369 \\
0,212\end{array}$ & $\begin{array}{l}0,274 \\
0,153\end{array}$ & $\begin{array}{l}C_{G}=0,5 ; \text { passando os caudais a ser } 0,288 \\
\text { e } 0,160 \mathrm{~m}^{3} / \mathrm{s} \text {, respetivamente. }\end{array}$ \\
\hline RA1 & 1,140 & 0,05 & 1,020 & Subm. & 0,108 & $0,188\left({ }^{4 *}\right)$ & $\mathrm{C}_{\mathrm{G}}=0,7 ;$ passando o caudal a ser $0,186 \mathrm{~m}^{3} / \mathrm{s}$ \\
\hline ST1 & $\begin{array}{l}0,350 \\
0,270\end{array}$ & $\begin{array}{l}0,233 \\
0,233\end{array}$ & $\begin{array}{c}\left.--{ }^{* *}\right) \\
---\end{array}$ & $\begin{array}{l}\text { Livre } \\
\text { Livre }\end{array}$ & $\begin{array}{l}0,222 \\
0,177\end{array}$ & $\begin{array}{l}0,171 \\
0,115\end{array}$ & $\begin{array}{l}\mathrm{C}_{\mathrm{G}}=0,45 ; \text { passando os caudais a ser } \\
0,166 \text { e } 0,132 \mathrm{~m}^{3} / \mathrm{s}, \text { respetivamente. }\end{array}$ \\
\hline $\begin{array}{ll}\text { ST2 - D4 } \\
\text { D5 }\end{array}$ & $\begin{array}{l}0,600 \\
0,700 \\
0,600\end{array}$ & $\begin{array}{c}0,700\left({ }^{5 *}\right) \\
0,080 \\
0,154\end{array}$ & $\begin{array}{l}0,600 \\
0,550 \\
0,530\end{array}$ & $\begin{array}{l}\text { Sem ressalto } \\
\text { Subm. } \\
\text { Subm. }\end{array}$ & 0,152 & $\begin{array}{l}0,115 \\
0,091 \\
0,064\end{array}$ & $\begin{array}{l}\mathrm{C}_{\mathrm{G}}=0,35 ; \text { passando o caudal a ser } 0,091 \mathrm{~m}^{3} / \mathrm{s} \\
\mathrm{C}_{\mathrm{G}}=0,35 ; \text { passando o caudal a ser } 0,071 \mathrm{~m}^{3} / \mathrm{s}\end{array}$ \\
\hline
\end{tabular}

(*) canal coberto a jusante - impossível usar o equipamento SRS5

$(* *) \quad$ Escoamento sempre livre por jusante

(3*) Comporta de derivação para o reservatório; diferença dos valores medidos pelos SRS5 e SIQ no Distribuidor 18, que alimenta o R18, respetivamente, a montante e a jusante da derivação

(4*) Comporta de derivação para o reservatório; diferença dos valores medidos pelo SRS5 no Canal Direito, que alimenta o RA1, respetivamente, a montante e a jusante da derivação

(5*) Comporta acima da superfície livre; caudal não controlado nem por comporta nem por soleira. 


\section{Relatório da Medição}

\section{Detalhes do Local}

Nome do Local

Código da Seção

Localização

\begin{tabular}{|lc|}
\hline \multicolumn{2}{|l|}{ Informações do Sistema } \\
\hline Tipo do Sistema & RS-S5 \\
Número de Série & 4000 \\
Versão do Firmware & 3.00 \\
Versão do Software & 3.6 .0 .3384 \\
\hline
\end{tabular}

Configurações do Sistema

\section{Prof. dos Transdutores ( $m$ )}

Salinidade (ppt)

Declinação Mag. (graus)
Data Medido: quarta-feira, 24 de setembro de 2014

Informações da Medição

Participantes

Barco/Motor

No da Medição

\begin{tabular}{llll}
\hline \multicolumn{2}{c}{ Configurações da Medição } & & \\
\hline Ref. para Trajeto & Bottom-Track & Método Margem Esq. & Margem Gradual \\
$\begin{array}{l}\text { Ref. para Prof. } \\
\text { Sist. de Coord. }\end{array}$ & Feixe Vertical & Método Margem Dir. & Margem Gradual \\
& & Tipo Extrapolação Superf. & Lei Exponêncial \\
& & Tipo Extrapolação Fundo & Lei Exponêncial \\
\hline
\end{tabular}

\begin{tabular}{|c|c|c|c|c|c|c|c|c|c|c|c|c|c|c|c|c|}
\hline \multirow{2}{*}{\multicolumn{17}{|c|}{ Resultados das Medições }} \\
\hline & & & & & & & & & & & & & & & & \\
\hline $\begin{array}{l}\text { No }^{2} \\
\text { da }\end{array}$ & \multicolumn{2}{|c|}{ Hora } & \multicolumn{4}{|c|}{ Dist. } & \multicolumn{3}{|c|}{ Vel. Méd. } & \multicolumn{6}{|c|}{ Vaz. } & \multirow{2}{*}{\begin{tabular}{|c}
$\%$ \\
Medidc \\
\end{tabular}} \\
\hline$\#$ & Hora & Duração| & Temp. & Trajeto & DMG & Larg. & Área & Emb. & Água & Esq. Dir. $\mathrm{s}$ & Superf. & Meio & Fundo & Total & LCTotal & \\
\hline $1 \mathrm{M}$ & 15:02:58 & $0: 01: 05$ & 18,9 & 2,67 & 2,12 & 2,835 & 1,432 & 0,041 & 0,141 & \begin{tabular}{|l|l|}
0,00 & 0,00 \\
\end{tabular} & 0,04 & 0,11 & 0,05 & 0,201 & - & 55,1 \\
\hline $2 \mathrm{M}$ & 15:04:26 & $0: 01: 04$ & 18,9 & 2,59 & 1,60 & 2,319 & 1,280 & 0,040 & 0,170 & \begin{tabular}{|l|l|}
0,00 & 0,01 \\
\end{tabular} & 0,04 & 0,12 & 0,05 & 0,217 & - & 53,4 \\
\hline $6 \mathrm{M}$ & 15:09:16 & $0: 01: 07$ & 18,8 & 2,52 & 1,81 & 2,532 & 1,258 & 0,038 & 0,174 & \begin{tabular}{|l|l|l|}
0,00 & 0,00 \\
\end{tabular} & 0,05 & 0,12 & 0,05 & 0,220 & -- & 53,2 \\
\hline & & Média & 18,9 & 2,59 & 1,84 & 2,562 & 1,323 & 0,040 & 0,162 & \begin{tabular}{l|l|}
0,00 & 0,00 \\
\end{tabular} & 0,04 & 0,11 & 0,05 & 0,213 & 0,000 & 53,9 \\
\hline & & $\begin{array}{l}\text { Desvio } \\
\text { Padrão }\end{array}$ & 0,1 & 0,06 & 0,21 & 0,212 & 0,077 & 0,002 & 0,015 & \begin{tabular}{l|l|}
0,00 & 0,00
\end{tabular} & 0,00 & 0,00 & 0,00 & 0,008 & 0,000 & 0,9 \\
\hline & & cv & 0,0 & 0,024 & 0,115 & 0,083 & 0,058 & 0,038 & 0,093 & 0,000 & 0,028 & 0,022 & 0,042 & 0,038 & 0,000 & 0,016 \\
\hline \multicolumn{17}{|c|}{ Tempo de Exposição: 0:03:16 } \\
\hline & 2.2 & 1145838r.rivr & & & & & & & & & & & & & & \\
\hline
\end{tabular}

\section{Coment.}

No da trav.20140924145838r.rivr - ; No da trav.20140924150006r.rivr - ; No da trav.20140924150455r.rivr - ;

Calibração da Bússola

Calibração com sucesso

Duração da calibração $=120 \mathrm{~s}$

M2.00 = Influência magnética tolerável

Q9 = Campo magnético é uniforme

H9 = Rotação horizontal completa

V6 $=$ Pitch/Roll Elevados

\section{Recomendações:}

Evite mudanças na configuração e orientação entre o sistema e as influências magnéticas detectadas durante a calibração da bússola.

A localização da travessia deve ter as mesmas propriedades magnéticas de onde a bússola foi calibrada.

\section{Testar Sistema}

Resultado: Sistema está operando normalmente

Parâmetros e configurações marcadas com um * não são constantes para todos os arquivos.

Figura 9. Estimativa do caudal com o caudalímetro SRS5 na estação D12 do SCADA, a jusante das comportas. 


\section{CONSIDERAÇÕES FINAIS}

O artigo apresenta os trabalhos de calibração de campo dos controladores de caudal para comportas instalados no SCADA do AHIN e os respetivos resultados.

A calibração de campo foi efetuada usando dois tipos diferentes de caudalímetros que que se baseiam no efeito de Doppler para determinar a velocidade da corrente. O coeficiente teórico de vazão usado no controlador de caudal foi, deste modo, ajustado a cada instalação

passando a ter-se em conta as diferentes situações de campo. Esta metodologia permitiu ainda a verificação do algoritmo geral de vazão usado para diferentes situações de funcionamento das estruturas soleiracomporta.

A experiência de campo obtida permite concluir que os caudalímetros SIQ fixos instalados são muito sensíveis ao estado de limpeza das cabeças dos respetivos sensores, recomendando-se, pelo menos, uma limpeza por semana. O sistema de montagem usado permite a fácil elevação do equipamento para limpeza.

\section{AGRADECIMENTOS}

Os autores agradecem à ARBI a disponibilização dos elementos de projeto para o presente artigo.

\section{BIBLIOGRAFIA}

Baume, J.P. et al. (2000) - Simulation of Irrigation Canals (SIC, version 3.7): Theoretical Concepts Modelling Approach. Volume II. Irrigation Division of CEMAGREF. Montpellier. France.

ITRC (2004) - Bottom Mounted Doppler Flow Meters for Canals, ITRC-Irrigation Training and Research Center, CALPOLY, CA, USA.

Rijo, M. (2010) - Canais de Adução. Projecto, Operação, Controlo e Modernização. 531p., Edições Sílabo, Lisboa, Portugal. ISBN: 978-972618-615-1.

Rijo, M., Honrado, J., Almeida, M. e Arranja, C. (2007) - Controlo de um canal de rega. Parte I - Definição e apresentação geral. Recursos Hídricos (Lisboa), Vol. 28(2), 5-15.

Rijo, M., Inácio, B. e Campos, J. (2016) Supervisão e controlo de um sistema de canais de rega. Parte I - Definição e apresentação geral. Recursos Hídricos (Lisboa) Vol. 37(1):59-72.

SonTek 2012 SonTeK IQ Intelligent Flow User's Manual, SonTeK, San Diego, CA, USA.

SonTek 2010 SonTeK RiverSurveyor S5/M9 System Manual, SonTeK, San Diego, CA, USA. 